
\title{
Research S Suare \\ Clinical Validity of the Smallest Oblique Sagittal Area of the Neural Foramen in Patients With Suspected Cervical Spondylotic Radiculopathy
}

\section{Yukun Jia}

The Second School of Clinical Medicine,Southern Medical University;Affiliated Baoan Hospital of

Shenzhen,Southern Medical Universaity

\section{Zhan Peng}

Affiliated Baoan Hospital of Shenzhen,Southern Medical University

\section{Yuantian Qin}

Nanjing University of Aeronautics and Astronautics

\section{Jin Li}

Affiliated Baoan Hospital of Shenzhen,Southern Medical University

\section{Guangye Wang ( $\sim$ doctorwang521@163.com )}

The Second School of Clinical Medicine,Southern Medical University;Affiliated Baoan Hospital of Shenzhen,Southern Medical University

\section{Research article}

Keywords: cervical spondylotic radiculopathy, neural foramen, stenosis, diagnostic imaging

Posted Date: August 9th, 2021

DOl: https://doi.org/10.21203/rs.3.rs-772499/v1

License: (1) This work is licensed under a Creative Commons Attribution 4.0 International License. Read Full License 


\section{Abstract}

Objective To evaluate the smallest oblique sagittal area of the neural foramen in detecting cervical spondylotic radiculopathy and to determine its potential significance for treatment decisions.

Methods The subjects of the study were patients with cervical spondylotic radiculopathy who visited the spine surgery from 2016 to 2019. All patients were compared according to the minimum oblique sagittal area and the cut-off point value, and they were divided into positive and negative parameters. The changes in neck disability index (NDI), Japanese Orthopaedic Association score (JOA) and visual analog scale (VAS) during the two treatment groups from baseline to at least 24 months of follow-up were compared.

Results In the surgery group, there was no significant difference in symptom improvement between patients with positive and negative parameters. In the non-surgical group, for patients with positive parameters, NDI decreased by 2.35 , JOA increased by 0.88 , and neck VAS score improved by 0.42 . For patients with negative parameters, NDI decreased by 10.32 , JOA increased by 2.86 on average, and neck VAS score improved by 2.46 points on average (both $\mathrm{p} \otimes 0.01$ on $\mathrm{t}$ test).

Conclusions In patients undergoing surgery, the symptoms of the patients have been significantly improved after surgery, and the smallest oblique sagittal area of the neural foramen seems to be unable to predict the outcome of the surgery. However, in non-surgical patients, the improvement of symptoms in the stenosis group was more limited. This may imply that surgery may be effective for patients who had positive parameters.

\section{Introduction}

Cervical spondylotic radiculopathy (CSR) is the most common type of cervical spondylosis ${ }^{1}$. In recent years, many studies have evaluated the clinical, imaging and other diagnostic criteria of CSR for better diagnosis and treatment ${ }^{2-4}$. However, a major problem is that the imaging results are not always consistent with clinical symptoms. In the treatment of CSR, there is a lack of high-quality evidence for the efficacy of conservative treatment and surgical treatment ${ }^{5}$, the scientific evidence for surgical indications is limited ${ }^{6-8}$. Guidance to clinicians is also inconsistent and inadequate.

In addition, the debate about which diagnostic criteria could help decide whether to adopt conservative treatment or surgical treatment and the choice of specific surgical procedures has been ongoing ${ }^{9-11}$. There are many randomized controlled trials comparing the efficacy between surgery and conservative treatment of $\mathrm{CSR}^{12 ; 13}$. Some studies have reported the effectiveness of surgery in relieving symptoms (pain relief, satisfaction, muscle weakness improvement) has been shown to be between $80 \%$ and $95 \%{ }^{14-17}$. However, some systematic reviews have found no clear benefits of surgery over nonsurgical treatments ${ }^{18 ;}{ }^{19}$. The evidence that can be drawn from these studies is considered to be limited. Results suggest that selection criteria, observer bias, the natural course and placebo mechanisms play an important role in the reported high success rates after surgery. Although the evidences for the benefits of decompression surgery is growing, the uncertainty around the indication for surgery remains. It is not clear which patients benefit most from each strategy. 
Recently, the study of Hong et al. ${ }^{20}$ showed that nerve root compression is mainly related to the decrease in the width and area of the neural foramen. Ozaki ${ }^{21}$ measured 1152 cases of cervical neural foramen in the oblique sagittal plane in 2019 , and the report pointed out that the best cut-off value of the minimum width of the nerve root canal to diagnose cervical nerve foramen stenosis is $2.7 \mathrm{~mm}$. It has certain reference value for the diagnosis of neural foraminal stenosis. Previous research ${ }^{22}$ has shown that the smallest oblique sagittal area of the cervical neural foramen can contribute to diagnose cervical radiculopathy caused by foraminal canal bony stenosis. A cut-off value of $25.95 \mathrm{~mm}^{2}$ showed relatively high sensitivity $(74.1 \%)$ for detecting bony stenosis of the neural foramen and good specificity (80.9\%). The next step of the research is to measure and evaluate the clinical value of the new parameters in a representative patient population to prove whether the smallest oblique sagittal area of the neural foramen can identify patients suspected of CSR, and help determine which patients are suitable for surgery or conservative treatment

There is no recognized diagnostic standard for $\mathrm{CSR}^{23}$, when there is no perfect reference standard, the ideal research should be to evaluate the correlation between the measurement result and its success of the operation ${ }^{24}$. We designed a retrospective study of the clinical effectiveness of the smallest oblique sagittal area of the neural foramen. The purpose of this study was to determine whether this parameter can provide valuable clinical information to help formulate a treatment plan (surgery or conservative treatment). We studied the relationship between changes in parameters before and after surgery or non-surgical treatment and changes in patient symptoms.

\section{Material And Methods}

This study was approved by the local ethics committee (BLY20190712). All patient data were anonymous and confidential. Verbal informed consent was obtained from participants. In brief, we evaluated the ability of this indicator to diagnose patients with suspected CSR by studying the correlation between the smallest oblique sagittal area of the neural foramen and the clinical results.

The eligibility assessment of patients who referred to our spine department was conducted between January 2016 and December 2019. Based on medical history and physical examination, including patients suspected of having CSR. The exclusion criteria are as follows: (1) Cervical neural foramen stenosis is caused by soft tissue, such as a herniated disc or ligament, rather than bone structure. (2) Cervical spine instability; (3) Cervical spine surgery history; (4) History of any neurological disease; (5) Tumor/malignant tumor; (6) Cause of trauma; (7) Congenital or other skeletal dysplasia.

The subjects underwent routine CT(Fig. 1) and images (slice thickness:1.000mm, slice increment:0.500mm; model: Optima; manufacturer: USA) of the cervical vertebrae in the neutral position were taken from C4 to T1. Then, use Mimics software to reconstruct the cervical spine and neural foramina model through 3D digital simulation (version: 20.0 Belgium Materialise) (Fig. 2). Determine the maximum plane of the intervertebral space on the coronal plane, and then make a midline of the target vertebral body on this plane, and make a straight line $45^{\circ}$ to the midline through the nerve foramen, which is the centerline of the nerve foramen. Then, cross the center line to make a section that intersects the reconstructed neural foramen, with an interval of 0.1 $\mathrm{mm}$. Finally, take the cross section with the smallest area (Fig. 3). This was considered to be the smallest oblique sagittal area of the neural foramen. These processes take about 45 minutes. 
Researchers collect patient clinical information from the medical record system, including patient medical history and self-assessment (Neck Disabilitv Index (NDI) ${ }^{25}$, Japanese Orthopaedic Association Scores (JOA),visual analog scale (VAS) for neck and arm pain), physical examination, and imaging (CT,MRI), and treatment programs (surgery or nonsurgical treatment). And the researchers also collected the patient's clinical follow-up information. Two other investigators independently reconstructed all imaging data retrospectively and measured all neural foramen sizes on 3-matic in the absence of clinical or surgical information. All surgeons received one-on-one guidance to ensure that they were familiar with the software program and that the measurements were consistent. They were not treating physicians of study patients and were blinded to all other clinical information. Before further analyses, discordant initial the measured results (the difference is more than $3 \mathrm{~mm}^{2}$ ) of the two investigators were resolved in a consensus meeting. Take the average of two measurements as the final calculated value. All data were entered into SPSS software to blind investigators to other study information.

All patients were compared according to the smallest oblique sagittal area of the neural foramen and the value of the critical point $\left(25.95 \mathrm{~mm}^{2}\right)$, and then divided into the parameter positive and negative.

The main outcome indicators were the changes in NDI, JOA and pain VAS between baseline and follow-up examinations. The changes in these efficacy indicators were compared between surgery group and non-surgery group. In the surgery group, patients who had no planned surgery initially but who underwent delayed decompression surgery during the follow-up period were evaluated. Secondary results include statistics on the rate of delayed surgery, as well as an evaluation of the consistency of the measurement results of the two investigators.

All data were statistically analysed using SPSS 20.0 (PASW Statistics, IBM). In the two treatment groups, we tested for a difference in the mean within-patient change in NDI, JOA and VAS between the stenosis and nonstenosis groups using Student $t$ test. The Kappa value was calculated to assess the inter-ethnic consistency between the measurement results of the two researchers. The statistical significance level used was $5 \%$.

\section{Results}

\section{patients}

A total of 199 patients with suspected CSR met the inclusion criteria. Among them, 31 cases were not followed up within the specified time interval, and 12 cases had to be excluded due to lack of result values. In total, 159 patients (mean age: 54 years, male: female =93:66) were included in the study, as detailed in Table 1. 
Table 1

Baseline patient characteristics in patients planned for surgery and no surgery

\begin{tabular}{|llll|}
\hline Characteristic & surgery $(\mathbf{n}=\mathbf{8 5})$ & Delayed surgery $(\mathbf{n = 1 3})$ & No surgery $(\mathbf{n = 6 1})$ \\
\hline Sex(M:F) & $55: 30$ & $5: 8$ & $33: 28$ \\
\hline Age(years) & & & \\
\hline Median & 52 & 43 & 51 \\
\hline Range & $23-81$ & $27-46$ & $25-88$ \\
\hline VAS neck pain & $6.82 \pm 1.15$ & $6.81 \pm 0.75$ & $5.83 \pm 0.72$ \\
\hline VAS arm pain & $6.19 \pm 0.91$ & $7.02 \pm 1.00$ & $5.49 \pm 1.15$ \\
\hline Loss of strength in arms & $29 \%$ & $33 \%$ & $15 \%$ \\
\hline Paresthesia & $49 \%$ & $52 \%$ & $33 \%$ \\
\hline JOA & $7.58 \pm 1.98$ & $7.92 \pm 1.38$ & $8.23 \pm 1.38$ \\
\hline NDI & $44.43 \pm 7.58$ & $45.28 \pm 10.98$ & $37.65 \pm 6.64$ \\
\hline VAS, visual analog scale; JOA, Japanese Orthopaedic Association Scores & \\
\hline
\end{tabular}

After the baseline assessment, 85 patients (53\%) underwent surgery. The median time from baseline assessment to surgery was 1.6 months. Of the remaining 74 patients, no surgical treatment was chosen. Their treatment generally included physical therapy and oral pain medication (nonsteroidal analgesic and neurotrophic drug $)^{26}$. Of the 74 patients who did not initially choose surgery, 13 of them were revised during the follow-up period. These patients received surgery, and the median time interval between delayed surgery and baseline assessment was 8.3 months (Fig. 4).

All procedures were performed by the same team of physicians, with anterior cervical discectomy and fusion. Table 1 lists the baseline characteristics of the included patients.

\section{Outcomes}

Table 2 lists the baseline value and prognosis improvement of the two treatment groups. The change from baseline to follow-up is shown in Fig. 5-8. Almost all patients who underwent surgery improved after treatment, while the results of the non-surgical group were poor. Among 98 patients in the surgical group, 59 were parameter-positive and 39 were parameter-negative. Patients with parameter-positive NDI decreased by an average of 26.52 points, JOA increased by an average of 6.88 points, and VAS neck pain improved by an average of 4.18. Patients with negative parameters decreased their NDI by an average of 23.79 points, JOA improved by an average of 6.33 points, and VAS neck pain improved by an average of 3.97 points (Table 2). 
Table 2

VAS, JOA and NDI measures according to sedimentation sign result, stratified by treatment group

\begin{tabular}{|c|c|c|c|c|c|c|c|c|}
\hline \multirow{2}{*}{$\begin{array}{l}\text { Treatment } \\
\text { group }\end{array}$} & \multicolumn{3}{|c|}{ Parameter positive } & \multicolumn{3}{|c|}{ Parameter negative } & \multirow{2}{*}{$\begin{array}{l}\text { Difference } \\
(95 \% \mathrm{Cl})\end{array}$} & \multirow{2}{*}{ value } \\
\hline & Baseline & $\begin{array}{l}\text { Follow- } \\
\text { up }\end{array}$ & Change & Baseline & $\begin{array}{l}\text { Follow- } \\
\text { up }\end{array}$ & Change & & \\
\hline \multicolumn{9}{|l|}{$\begin{array}{l}\text { Surgical } \\
\text { group }\end{array}$} \\
\hline $\begin{array}{l}\text { VAS neck } \\
\text { pain }\end{array}$ & 7.09 & 2.91 & 4.18 & 6.40 & 2.67 & 3.72 & $0.46(-0.04,0.96)$ & 0.71 \\
\hline $\begin{array}{l}\text { VAS arm } \\
\text { pain }\end{array}$ & 6.46 & 2.35 & 4.11 & 6.06 & 2.09 & 3.97 & $0.14(-0.41,0.70)$ & 0.61 \\
\hline JOA & 6.92 & 13.80 & -6.88 & 7.87 & 14.20 & -6.33 & $\begin{array}{l}-0.21 \\
(-1.22,0.80)\end{array}$ & 0.68 \\
\hline NDI & 46.78 & 20.26 & 26.52 & 41.16 & 17.37 & 23.79 & $2.73(-0.57,6.3)$ & 0.10 \\
\hline \multicolumn{9}{|c|}{$\begin{array}{l}\text { Nonsurgical } \\
\text { group }\end{array}$} \\
\hline $\begin{array}{l}\text { VAS neck } \\
\text { pain }\end{array}$ & 5.94 & 5.52 & 0.42 & 5.74 & 3.29 & 2.46 & $-0.5(-1.1,0.0)$ & $<0.01$ \\
\hline $\begin{array}{l}\text { VAS arm } \\
\text { pain }\end{array}$ & 5.98 & 5.12 & 0.86 & 5.08 & 2.91 & 2.17 & $0.2(-0.2,0.5)$ & $<0.01$ \\
\hline JOA & 7.92 & 8.81 & -0.88 & 8.46 & 11.34 & -2.89 & $0.2(-0.6,0.9)$ & $<0.01$ \\
\hline NDI & 39.89 & 37.54 & 2.35 & 35.99 & 25.67 & 10.32 & $-0.9(-2.5,0.7)$ & $<0.01$ \\
\hline
\end{tabular}

Among the 13 patients with delayed surgery, 5 patients were positive parameters and 8 patients were negative parameters. The median baseline NDI was 45.28 , and the JOA was 7.92. Overall, the average NDI improved after delayed surgery was 25.77 , JOA improved by 6.33 after delayed surgery, neck pain improved by 3.91 , and arm pain improved by 4.88 . There was no statistically significant difference between these changes between positive and negative parameters.

Among 61 patients in the non-surgical group, 26 patients were positive parameters and 35 were negative parameters. For patients with positive parameters, NDI decreased by 2.35 on average, JOA increased by 0.88 on average, and neck VAS score improved by 0.42 on average. For patients with negative parameters, NDI decreased by 10.32 , JOA increased by 2.86 on average, and neck VAS score improved by 2.46 points on average (Table 2).

Baseline test results for patients with positive and negative parameter are shown in Table 3. patients with positive often had more functional limitations in the NDI at baseline than patients with negative parameters. A loss of arm strength was more common in the patients with positive; paresthesia in the arm was more prevalent in the patients with positive. Regardless of the smallest oblique sagittal area of the neural foramen, 
similar neck and arm pain levels were observed. $51 \%$ of patients in the patients with positive indicated their preference for decompression surgery, whereas $38 \%$ of those in the patients with negative parameters, preferred nonsurgical treatment.

Table 3

Comparison of sedimentation sign results with other diagnostic tests at baseline

\begin{tabular}{|lll|}
\hline Test & Parameter positive $(\mathbf{n}=\mathbf{8 5})$ & Parameter negative $(\mathbf{n}=\mathbf{7 4})$ \\
\hline VAS neck pain & $6.74 \pm 1.20$ & $6.09 \pm 0.81$ \\
\hline VAS arm pain & $6.31 \pm 1.01$ & $5.60 \pm 1.10$ \\
\hline JOA & $7.59 \pm 1.44$ & $8.16 \pm 2.01$ \\
\hline NDI & $44.67 \pm 8.33$ & $38.71 \pm 6.91$ \\
\hline Loss of strength in arms & $24 \%$ & $18 \%$ \\
\hline Paresthesia & $38 \%$ & $31 \%$ \\
\hline NDI, Neck Disabilitv Index; VAS, visual analog scale. JOA, Japanese Orthopaedic Association Scores \\
\hline * Values are expressed as median \pm Standard deviation \\
\hline
\end{tabular}

The two spine surgeons who used CT data to reconstruct the neural foramen and measure the minimum crosssectional area agreed in 149 (93.7\%). Both considered 79 patients as parameter-positive and 70 patients as parameter-negative. The resulting kappa coefficient was $0.874 \pm 0.039$.

\section{Discussion}

This study evaluated the clinical significance of the smallest oblique sagittal area of the neural foramen in detecting CSR. After the initial report of the parameter, which demonstrated its ability to detect CSR under ideal circumstances ${ }^{22}$, this study takes another step forward in the evaluation of parameters by evaluating the clinical significance of the smallest oblique sagittal area of the neural foramen in a wider group of patients. Among patients undergoing decompression surgery or non-surgical treatment, the changes in NDI, JOA, and pain between the patients with positive and negative parameters from baseline to 24 months follow-up were compared. Regardless of the parameters, the prognosis of patients in the surgery group was similar. In contrast, in the non-surgical treatment cohort, patients with positive parameters improved less than those with negative parameters. The Kappa values of the measurement data of the two researchers showed excellent agreement.

The most interesting result of this study was that the symptoms of patients with positive parameters did not improve after conservative treatment, while the symptoms of patients with negative parameters were relieved after conservative treatment. There were differences in symptom improvement between patients with negative and positive parameters after receiving conservative treatment, but there was no similar difference between patients undergoing surgery, indicating that patients with positive parameters may be more suitable for surgical treatment, or patients with positive parameters are not recommended to accept conservative treatment. In 
addition, patients with positive parameters had higher baseline NDI, lower JOA scores, and higher levels of neck and arm pain, indicating that CSR was more severe than patients with negative parameters.

In this study, the delayed operation rate was not related to the value of the smallest oblique sagittal area of the neural foramen. In addition to the clinical severity of CSR, other factors (such as the patient's economic level) may also play an important role in the initial surgical decision. In addition, other surgical indications, such as progressive neurological impairment, associated manifestations of cervical spondylotic myelopathy, segmental instability, and kyphosis, were the reasons for the subsequent decision of some of these patients to undergo surgery 27 .

The results of this study indicate that the smallest oblique sagittal area of the neural foramen provides useful information for the diagnosis of cervical spondylotic radiculopathy and helps identify patients who may benefit from surgery. Since almost all patients suspected of having CSR have undergone a CT examination, the measurement of this parameter does not cost extra, and its use is very simple and fast. In addition, in clinical practice, this parameter can clearly and visually convey the stenosis of the neural foramen to the patient. It is an absolute measure of neural foramen. We believe that this parameter can improve the clinical diagnosis of CSR.

Our study has some limitations. First, our study adopted a retrospective design. Second, it was a single-center study. This may limit the generalizability of our results. Multi-center prospective studies are needed to replicate and verify our results. Third, the time for follow-up examinations cannot be controlled. We accepted a follow-up interval of 22 to 31 months. A follow-up that is too short may not provide enough time to prove the benefits of the operation, while a follow-up that is too long may compromise the prognosis due to the degeneration of adjacent segments. Between 12 and 21 months after the baseline examination, we followed up most patients. In addition, after the initial postoperative improvement, the effect of the surgery did not seem to change over time, and a multivariate analysis of our data showed that changes in the follow-up interval did not have a significant effect on the results.

Further studies on the parameters of the smallest oblique sagittal area need to evaluate the applicability of our findings in different clinical settings. It is necessary to provide clinicians with more practical guidance, detailing how to interpret parameters in more complex situations (such as multi-level CSR). The clinical value of this indicator should also be evaluated in randomized controlled trials.

\section{Conclusion}

In patients who usually undergo decompression surgery, the smallest oblique sagittal area of the neural foramen does not seem to predict the outcome of the surgery. However, in conservatively treated patients, the smallest oblique sagittal area is less than the critical value and is associated with more limited symptom improvement. In these cases, surgery may be effective, but this needs to be investigated in prospective randomized trials.

\section{Declarations}

Ethics approval and consent to participate: 
informed consent was obtained from patient.

\section{Consent for publication:}

Proof of consent was obtained from study participants. all authors agree to publish.

\section{Authors' contributions:}

Y J: Formal analysis, Data curation, Writing- Original draft preparation, Writing- Reviewing and Editing.

Z P: Resources, Supervision, Writing- Reviewing and Editing.

$\mathrm{J}$ L: Validation.

J C: Writing- Reviewing and Editing

Y Q: Writing- Reviewing and Editing

G W: Conceptualization, Methodology, Project administration.

all authors have read and approved the manuscript

\section{Acknowledgements:}

no.

\section{Funding:}

no funding was received

Competing interests:

all authors declare that they have no conflict of interest.

\section{Availability of data and materials:}

Data was already included in the manuscript.

\section{References}

1. Yang F, Li WX, Liu Z, et al. 2016. Balance chiropractic therapy for cervical spondylotic radiculopathy: study protocol for a randomized controlled trial. Trials 17:513.

2. Kim S, Lee JW, Chai JW, et al. 2015. A New MRI Grading System for Cervical Foraminal Stenosis Based on Axial T2-Weighted Images. Korean journal of radiology 16:1294-1302.

3. Park HJ, Kim SS, Lee SY, et al. 2013. A practical MRI grading system for cervical foraminal stenosis based on oblique sagittal images. The British journal of radiology 86:20120515.

4. Park HJ, Kim SS, Han CH, et al. 2014. The clinical correlation of a new practical MRI method for grading cervical neural foraminal stenosis based on oblique sagittal images. AJR American journal of 
roentgenology 203:412-417.

5. Corey DL, Comeau D. 2014. Cervical radiculopathy. The Medical clinics of North America 98:791-799, xii.

6. Rhee JM, Yoon T, Riew KD. 2007. Cervical radiculopathy. The Journal of the American Academy of Orthopaedic Surgeons 15:486-494.

7. Eubanks JD. 2010. Cervical radiculopathy: nonoperative management of neck pain and radicular symptoms. American family physician 81:33-40.

8. Caridi JM, Pumberger M, Hughes AP. 2011. Cervical radiculopathy: a review. HSS journal : the musculoskeletal journal of Hospital for Special Surgery 7:265-272.

9. Carette S, Fehlings MG. 2005. Clinical practice. Cervical radiculopathy. The New England journal of medicine 353:392-399.

10. Taso M, Sommernes JH, Kolstad F, et al. 2020. A randomised controlled trial comparing the effectiveness of surgical and nonsurgical treatment for cervical radiculopathy. BMC musculoskeletal disorders 21:171.

11. Persson LC, Carlsson CA, Carlsson JY. 1997. Long-lasting cervical radicular pain managed with surgery, physiotherapy, or a cervical collar. A prospective, randomized study. Spine 22:751-758.

12. Engquist $M$, Löfgren $H$, Öberg $B$, et al. 2013. Surgery versus nonsurgical treatment of cervical radiculopathy: a prospective, randomized study comparing surgery plus physiotherapy with physiotherapy alone with a 2-year follow-up. Spine 38:1715-1722.

13. Engquist M, Löfgren H, Öberg B, et al. 2017. A 5- to 8-year randomized study on the treatment of cervical radiculopathy: anterior cervical decompression and fusion plus physiotherapy versus physiotherapy alone. Journal of neurosurgery Spine 26:19-27.

14. Hacker RJ, Cauthen JC, Gilbert TJ, et al. 2000. A prospective randomized multicenter clinical evaluation of an anterior cervical fusion cage. Spine 25:2646-2654; discussion 2655.

15. Moreland DB, Asch HL, Clabeaux DE, et al. 2004. Anterior cervical discectomy and fusion with implantable titanium cage: initial impressions, patient outcomes and comparison to fusion with allograft. The spine journal : official journal of the North American Spine Society 4:184-191; discussion 191.

16. Anderson PA, Sasso RC, Rouleau JP, et al. 2004. The Bryan Cervical Disc: wear properties and early clinical results. The spine journal : official journal of the North American Spine Society 4:303s-309s.

17. Nandoe Tewarie RD, Bartels RH, Peul WC. 2007. Long-term outcome after anterior cervical discectomy without fusion. European spine journal : official publication of the European Spine Society, the European Spinal Deformity Society, and the European Section of the Cervical Spine Research Society 16:1411-1416.

18. van Middelkoop M, Rubinstein SM, Ostelo R, et al. 2013. Surgery versus conservative care for neck pain: a systematic review. European spine journal : official publication of the European Spine Society, the European Spinal Deformity Society, and the European Section of the Cervical Spine Research Society 22:87-95.

19. Nikolaidis I, Fouyas IP, Sandercock PA, et al. 2010. Surgery for cervical radiculopathy or myelopathy. The Cochrane database of systematic reviews 2010:Cd001466.

20. Sohn HM, You JW, Lee JY. 2004. The relationship between disc degeneration and morphologic changes in the intervertebral foramen of the cervical spine: a cadaveric MRI and CT study. Journal of Korean medical science 19:101-106. 
21. Ozaki M, Nishioka K, Kitayama M, et al. 2020. Quantitative evaluation for cervical foraminal bony stenosis based on angled sagittal slices along a nerve root on computed tomography. Journal of clinical neuroscience : official journal of the Neurosurgical Society of Australasia 71:89-92.

22. Jia Y, Peng Z, Li J, et al. 2021. A New Parameter, the Smallest Oblique Sagittal Area of the Neural Foramen, as an Index to Diagnose Cervical Neural Foramen Stenosis. Canadian Association of Radiologists journal = Journal l'Association canadienne des radiologistes:8465371211005540.

23. Wainner RS, Gill H. 2000. Diagnosis and nonoperative management of cervical radiculopathy. The Journal of orthopaedic and sports physical therapy 30:728-744.

24. Reitsma JB, Rutjes AW, Khan KS, et al. 2009. A review of solutions for diagnostic accuracy studies with an imperfect or missing reference standard. Journal of clinical epidemiology 62:797-806.

25. Kavanagh C, Uy NS. 2019. Nephrogenic Diabetes Insipidus. Pediatric clinics of North America 66:227-234.

26. Lin T, Wang Z, Chen G, et al. 2020. Predictive Effect of Cervical Sagittal Parameters on Conservative Treatment of Single-Segment Cervical Spondylotic Radiculopathy. World neurosurgery 134:e1028-e1036.

27. Leveque JC, Marong-Ceesay B, Cooper T, et al. 2015. Diagnosis and Treatment of Cervical Radiculopathy and Myelopathy. Physical medicine and rehabilitation clinics of North America 26:491-511.

\section{Figures}
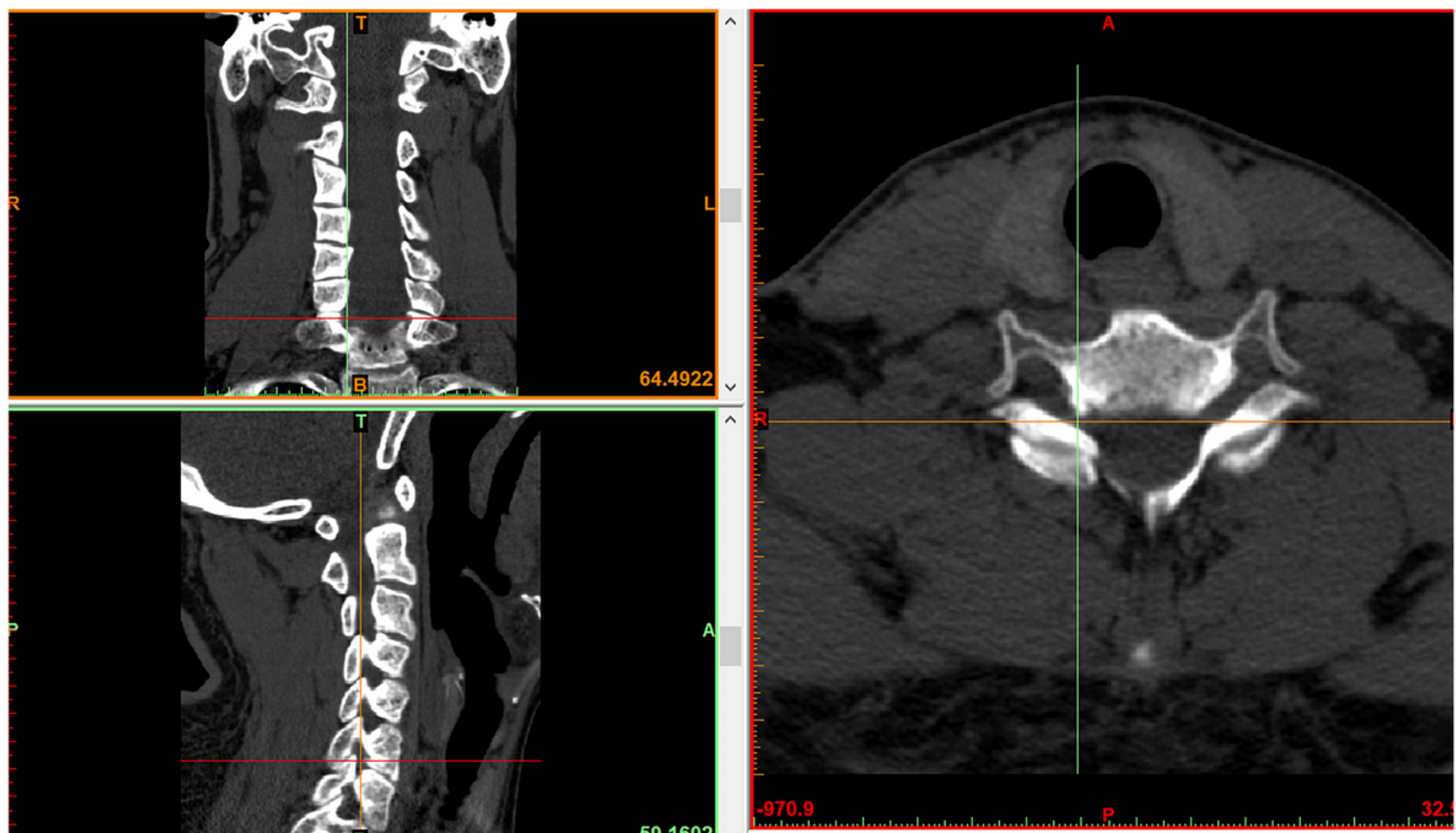

\section{Figure 1}

CT image of a patient's neural foramen stenosis. 


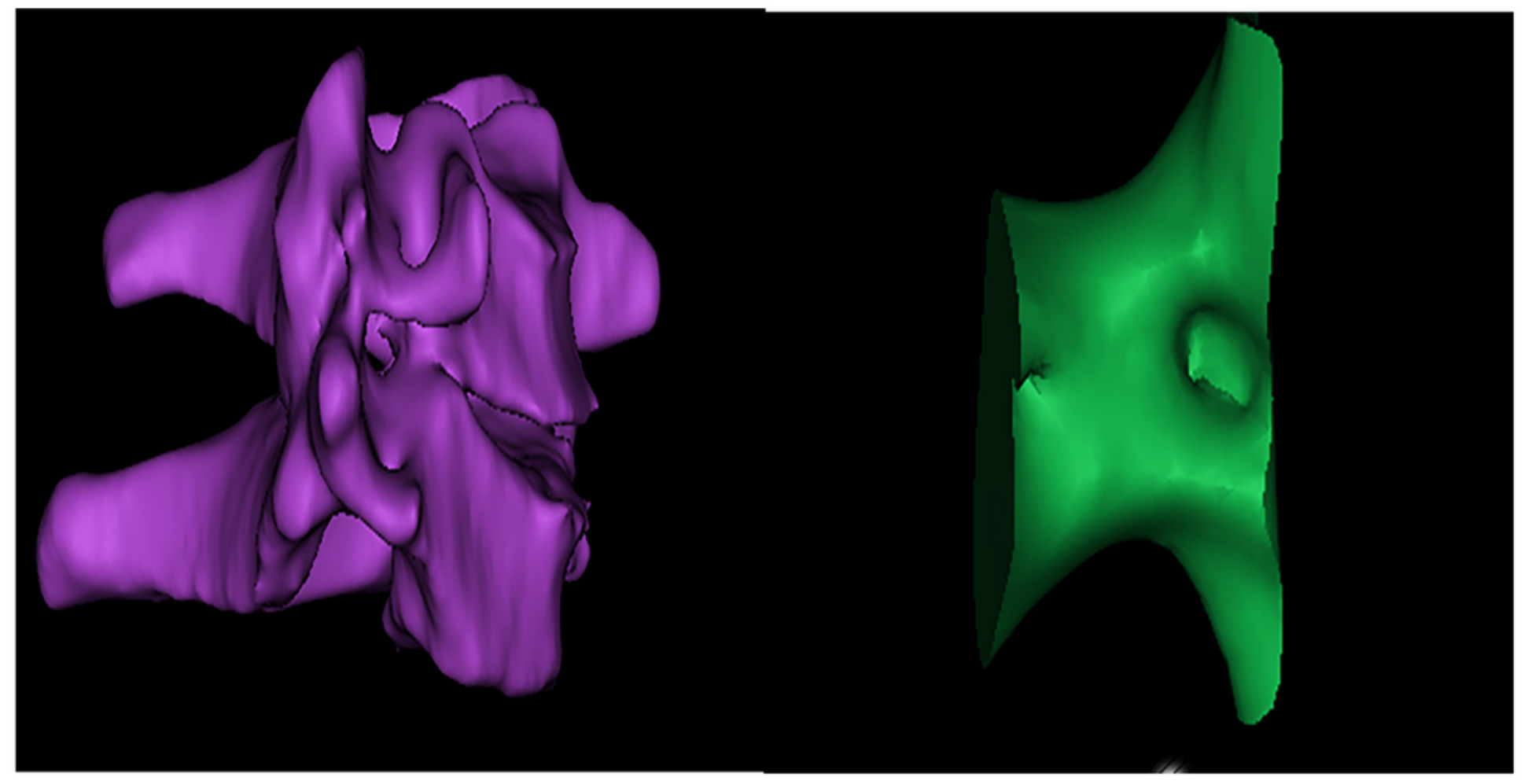

Figure 2

Three-dimensional digital simulation reconstruction model of cervical vertebra and neural foramen. 


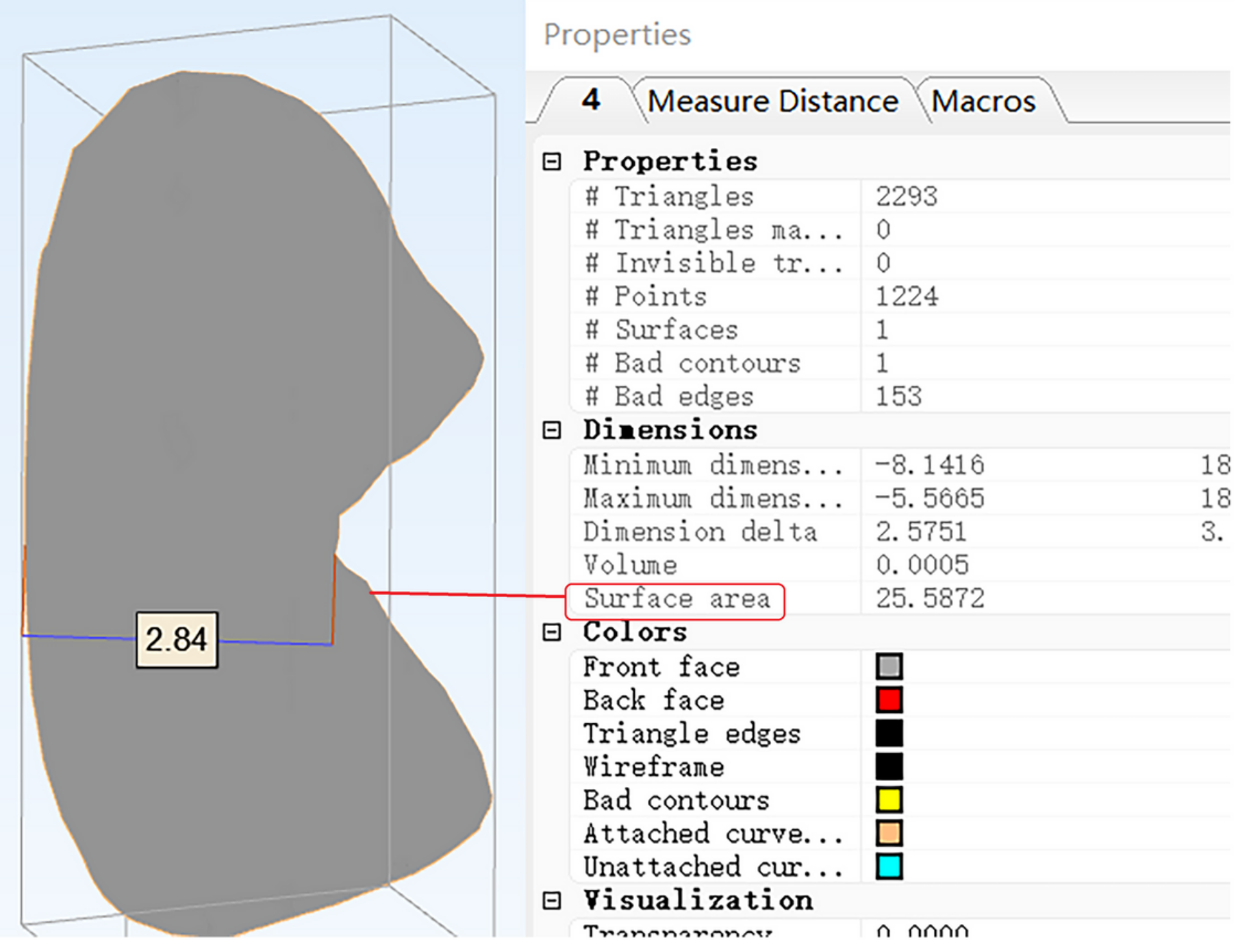

Figure 3

The smallest plane: The figure shows the measured value of the smallest oblique sagittal area of the neural foramen. 


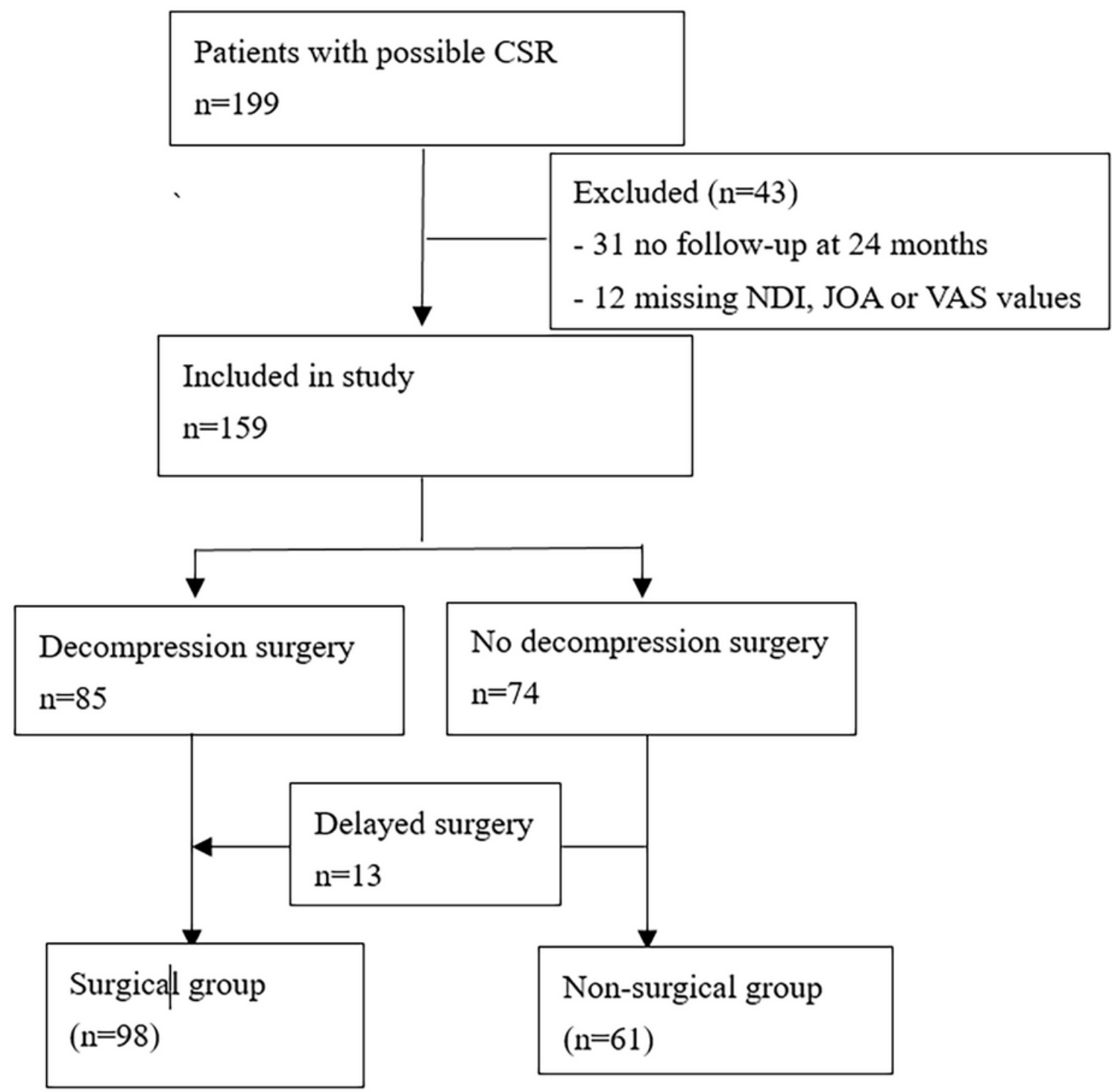

Figure 4

Participants' research profile 

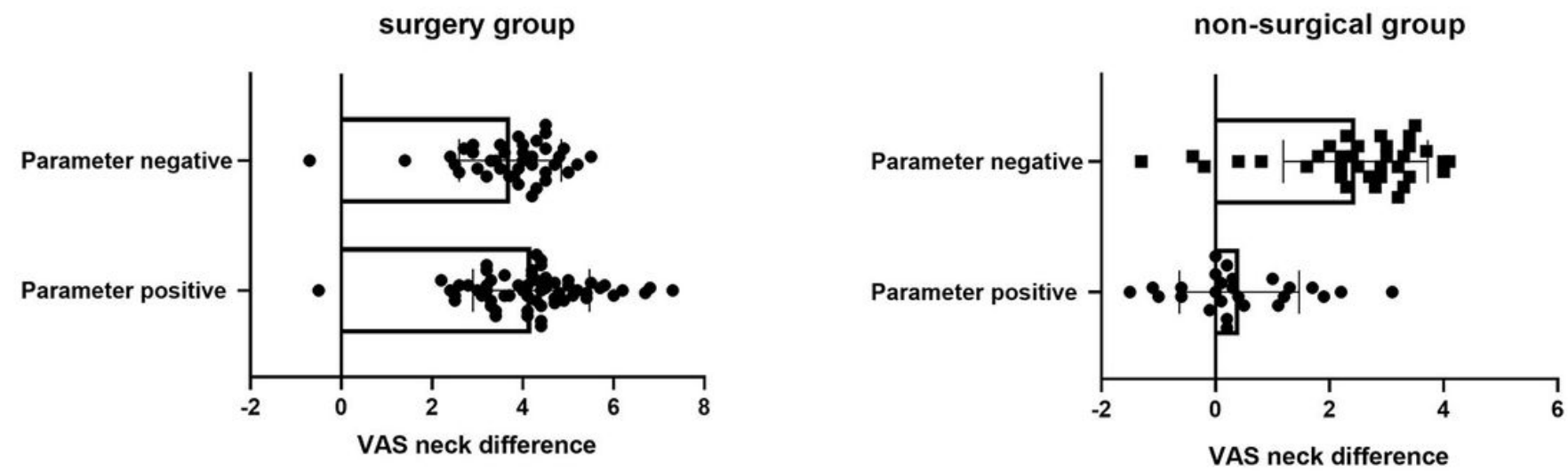

Figure 5

Change of VAS neck pain in treatment groups.
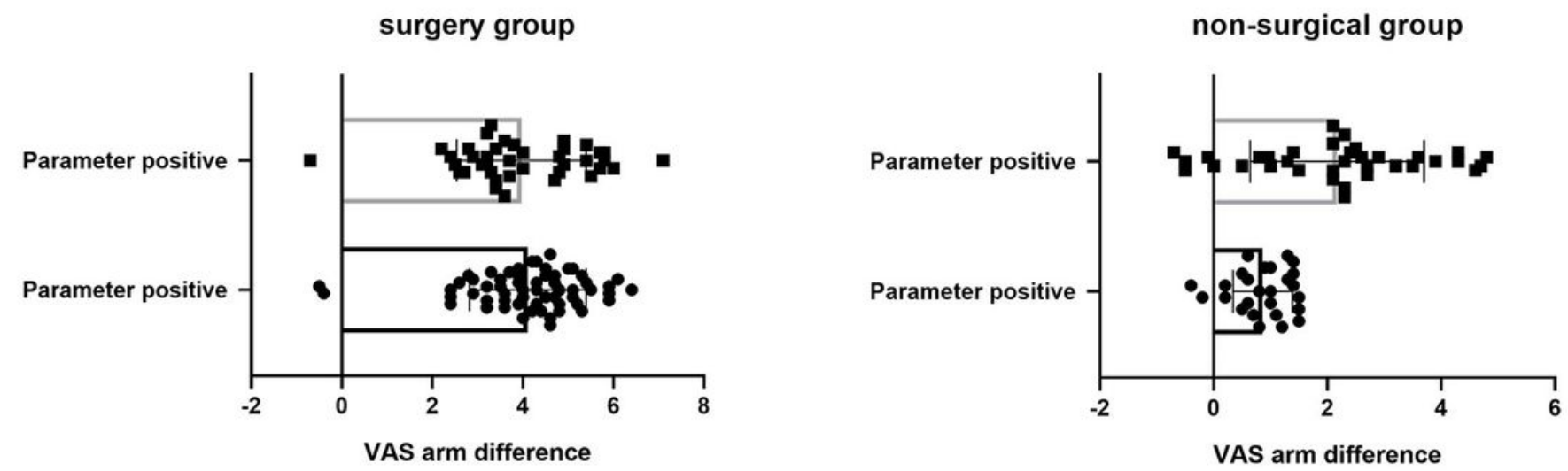

Figure 6

Change of VAS arm pain in treatment groups.
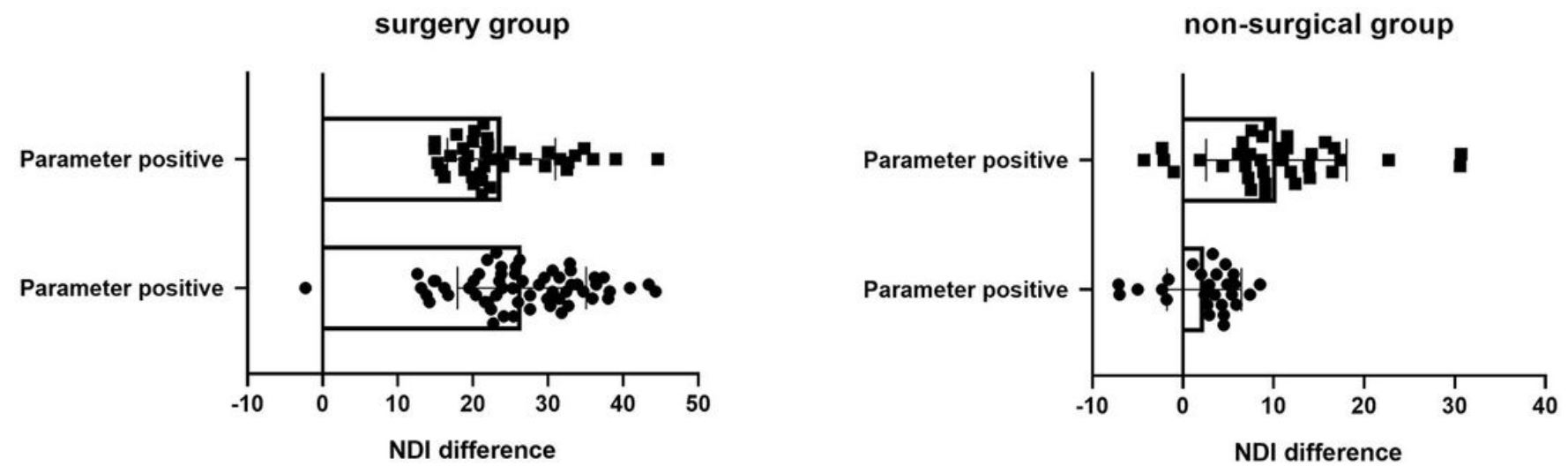

Figure 7 
Change of NDI in treatment groups.
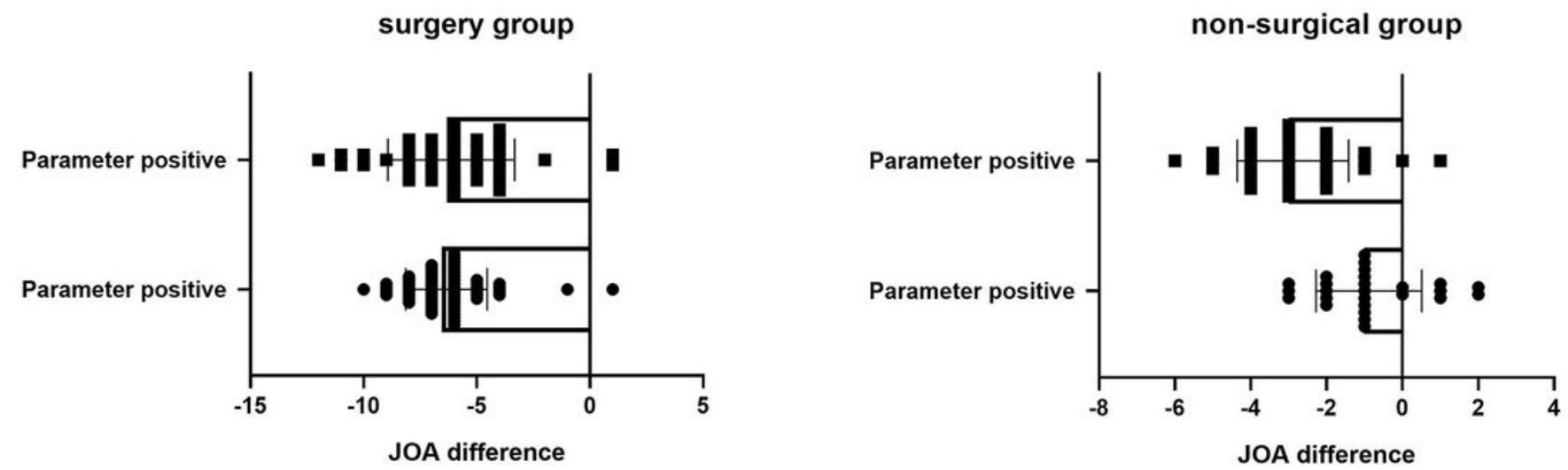

Figure 8

Change of JAO in treatment groups. 